\title{
PERMANENCES ET MUTATIONS DES ÉMISSIONS POLITIQUES EN FRANCE
}

\author{
Eric Darras ${ }^{1}$
}

« Sucer c'est tromper?» La question s'adresse à Michel Rocard, ancien premier ministre. Elle est formulée par un chef d'entreprise, Thierry Ardisson, animateur d'une émission qu'il produit pour être diffusée sur une chaîne du service public ${ }^{2}$. À ce point d'irrespect démocratique, une antienne bien connue semble définitivement confirmée : le discours politique s'effondre sous les assauts successifs d'un demisiècle de télévision. La victime - le politique - et le coupable - la télévision - sont clairement identifiés. Voici quelques autres évidences, extraites parmi les plus suffisantes de la littérature critique consacrée à la politique télévisée, dont le lecteur conviendra sans doute :

- «le spectacle est roi »;

- le temps des « grands rendez-vous politiques » n'est plus ;

- le «mélange des genres » s'est imposé ;

1 Eric Darras est maître de conférences à l'IEP de Toulouse.

2 Tout le monde en parle, France 2, 31 mai 2001. La presse écrite omet presque systématiquement de contextualiser la question qui s'explique par le genre de la séquence, par le contexte de «l'affaire Monica Lewinsky »; elle était elle-même précédée d'un questionnement rigoureux, bien qu'axé sur la biographie du « grand homme». Il est par ailleurs intéressant de constater que les réceptions scandalisées des journalistes de presse écrite tranchent avec celles de téléspectateurs de milieu populaire, qui y voient plus volontiers un amusement sans conséquence. Cf. Petit (Alexandra), Medias et opinions : réception de l'image des hommes politiques dans les émissions de divertissement, DEA de science politique, Université de Toulouse $1,2002$.

Recherches en communication, $\mathrm{n}^{\circ} 24$ (2005). 
- les hommes politiques sont « victimes » de la mise en scène télévisuelles de leur vie privée et jusqu'à leur intimité ;

- le discours politique « se raréfie » à la télévision ;

- le service public se plie aux logiques des chaînes privées ;

- les journalistes politiques ont cédé la place aux «citoyens lambda » et aux animateurs ;

- en conséquence, le débat politique se nivelle par le bas.

Chacune de ces affirmations est pourtant « fausse » ou insuffisante et au mieux indémontrable - pour ce qui concerne les moins clairement formulées, c'est-à-dire les moins empiriquement falsifiables -. Une affirmation aussi singulièrement flottante que «le spectacle est roi » à la télévision navigue entre le sans doute vrai et le même pas faux. Mais si l'on souhaite ainsi préciser plus clairement que le travail du réalisateur sur le plateau devient toujours plus signifiant (usages des plans de coupes, du film des mains, de la moitié inférieure du corps, surenchère sémiologique...) une mise en perspective historique relativise alors immédiatement une telle hypothèse de la «spectacularisation », sans la contredire tout à fait. Dans les années 60, les premiers magazines politiques de la télévision française font un usage rétrospectivement surprenant des possibilités de la caméra, ils ne s'interdisent pas de filmer les réactions en gros plan de l'homme politique d'opposition muet face aux questions parfois agressives des plus prestigieux journalistes de la presse écrite d'opinion, eux-mêmes anciens résistants, comme lorsque Roger Stéphane interroge François Mitterrand en faisant allusion à son passé vichyste dans Face à face en 1966: «Depuis quand vous considérez-vous comme un homme de gauche, M. Mitterrand ?»1.

Mais d'abord qu'est ce qu'une «émission politique »? Il n'existe pas véritablement de propriétés intrinsèques de l'émission politique, si ce n'est la participation régulière de professionnels de la politique. Il subsiste par contre un flou sur la définition de l'émission politique, luimême entretenu par les professionnels des médias. TF1 peut alternativement présenter 7 sur 7 comme une émission «d'actualité et surtout pas une émission politique » face au « grand public », ne serait-ce qu'en raison du caractère supposé déplaisant pour l'audience du qualificatif «politique », puis comme un programme politique lorsqu'il s'agit de vanter les réalisations de la chaîne auprès du CSA. La dilution du poli-

\footnotetext{
1 Face à face, 9 mai 1966.
} 
tique dans des formats plus vendeurs complexifie ainsi la compréhension du phénomène. Quoiqu'il en soit, le principal obstacle à une meilleure compréhension collective des enjeux «démocratiques» du traitement télévisuel des acteurs et institutions politiques réside précisément dans l'importation au sein de l'Université de la rhétorique du procès (ou du registre « pathologique ») qui, en cherchant des responsabilités simples, aveugle non seulement sur le constat (ou le « diagnostic ») mais aussi sur les causes plus structurelles de la transformation des rapports (de forces) entre professionnels de la politique et de la télévision.

Il convient de ne pas céder aux sirènes peu lucides d'un âge d'or de la politique télévisée. Il demeurerait introuvable en France. En fait de déclin, le discours politique se développe sous de nouvelles formes mais aussi, concurremment par une sorte de retour aux sources, via un renouveau des formats dont le face à face entre deux ou plusieurs hommes politiques.

Le déclin des programmes politiques relève largement de l'idée reçue. Rémi Festa, directeur des études de France Télévision, constate à partir des « piges Médiamétrie » que le temps consacré aux émissions politiques à la télévision sur les trois chaînes leaders ne fléchit guère que de 3,5\% entre 1991 et $2001^{1}$. Si, hélas, aucune statistique n'est disponible pour l'offre politique des chaînes du câble et du satellite, le discours politique à la télévision s'est inévitablement et considérablement accru en volume, une fois considérée la multiplication des fréquences. Toutefois, il ne s'agit ici que de la prise en compte de l'offre télévisuelle, et non de l'évolution de la consommation de programmes politiques étroitement dépendante de la chaîne et de place accordée aux émissions politiques dans sa grille de programmation. L'accroissement en volume de l'offre politique télévisuelle n'implique nullement un élargissement de l'accès du corps électoral à l'information politique ${ }^{2}$.

1 Le genre «information : émissions politiques»- donc hors JT et magazines d'informations - cumule 8739 heures en 1991 contre 7876 en 2001. Il faut ici volontairement exclure les années électorales et le découpage du corpus n'est certes pas optimal, il peut s'agit aussi ici de célébrer le service public (cf infra). Cf. Rémi Festa, «L'audience des émissions politiques », Dossiers de l'audiovisuel, n ${ }^{\circ} 102$, Paris, mars-avril 2002, p. 73.

2 Près des deux tiers des Français « regardent » le JT approximativement un soir sur deux au moins mais c'est principalement en raison d'une logique d'offre créant sa propre demande. L'heure de programmation comme le volume d'information offerts par les deux JT des deux chaînes leaders ( $20 \mathrm{~h}$ et plus de 30 minutes) ne peuvent s'expliquer par les logiques d'audience et relèvent d'une autocensure des 
Au contraire, les émissions politiques sont désormais nettement plus concurrencées par des programmes de divertissement autrement plus attractifs. Autrement dit, le coût de l'intérêt pour la politique augmente en conséquence. La baisse globale des audiences liée au déplacement dominical des programmes politiques sur le modèle américain ou en seconde partie de soirée s'est toutefois déjà effectuée en France au milieu des années 80 .

\section{Transformation des émissions politiques}

Enfin les émissions politiques se transforment. Face à la double contrainte des exigences politiques consignées dans le cahier des charges co-signé par le CSA d'une part, et de la maximisation de l'audience utile d'autre part, les programmes politiques :

- Se saisonnalisent d'une part autour des principales échéances électorales. Le temps d'antenne politique augmente sensiblement dans la perspective d'une élection majeure - le phénomène n'est certes pas nouveau ${ }^{1}$ mais s'accentue - tant pour des raisons éthiques et juridiques que pour des motivations commerciales (à mesure que les opinions politiques des téléspectateurs sont mobilisées par le débat public à l' approche de l'échéance, la politique devient plus et mieux «vendable »). C'est ainsi, d'autre part, que la saisonnalité prend désormais une autre forme avec les hard news ou breaking news en matière politique qui génèrent une audience considérable (émeutes dans les banlieues, grèves d'envergure, attentats...). Ces moments paroxystiques rapidement entretenus par des «pseudo-événements » (dont l'usage stratégique du sondage de l'émission politique pour multiplier les reprises le lendemain dans la presse) deviennent le prétexte d'une multiplication des émissions «spéciales » spécifiques ou ad hoc, autant de «forums politiques » d'autant plus nombreux qu'ils satisfont cette fois à la double exigence des leaders politiques et des annonceurs. Cette saisonnalité accentue le monopole de la parole politique télévisuelle sur les audiences majeures

programmateurs ou d'une tradition vraisemblablement menacée.

1 L'histoire des magazines politiques est inséparable de l'histoire électorale, dans les années 60 et 70 c'est à chaque fois en raison de l'approche d'une élection législative ou présidentielle que les professionnels réussissent à imposer au gouvernement un nouveau magazine qui du seul fait de sa périodicité fixe suppose l'accès de l'opposition à la télévision. Cf. Eric Darras, L'institution d'une tribune politique. Genèse et usages du magazine politique de télévision, Thèse de doctorat de science politique, Université de Paris II Panthéon-Assas, 1998, à paraître en 2006. 
(c'est-à-dire sur les chaînes leaders en prime time) dont bénéficie les leaders politiques de premier rang : le Président de la République, le Premier ministre et les principaux ministres, plus ceux " prévendus », selon la formule de Jay Rosen, par une actualité «brûlante » dont le ministre de l'intérieur (mais aussi Jean-Marie Le Pen) en période de trouble de l'ordre public, le ministre de l'Education nationale en cas de grève étudiante ou autre. Dans ces configurations politiques de crise, ils génèrent en effet non seulement plus d'audience mais aussi plus de retombées en presse écrite au bénéfice de l'image de la chaîne.

- Se fragmentent en séquences du journal télévisé, en particulier sur TF1 (avec la séquence Invité spécial) à l'approche des échéances électorales mais aussi sur France 3 (Dimanche soir) et France 2 (avec la séquence finale du JT Question ouverte présentée alternativement par Alain Duhamel, Olivier Mazerolle et Gérard Leclerc) comme au sein d'autres émissions omnibus (dont Les 4 vérités dans Télématin, et sur Canal Plus en matinée et en access prime time avec Nulle part ailleurs lors de sa reprise en main par Guillaume Durand). Cela permet de minimiser les pertes d'audience induites par la prise de parole politique hors actualité politique, tout en sacrifiant aux exigences contractuelles comme au contrôle du CSA et en entretenant une relation plus ou moins obligée avec les décideurs politiques les plus influents.

- Se concentrent sur le service public audiovisuel. Parmi les quatre chaînes généralistes leaders, les deux chaînes publiques assument $94 \%$ de l'expression politique totale hors campagne électorale en 2004 : la chaîne leader par l'audience TF1 ne consacre que $11 \mathrm{~h} 28$ minutes (et $2 \mathrm{~h} 47$ minutes seulement hors JT) à la parole politique entre le $1^{\text {er }}$ janvier et le 31 décembre 2004, pour 99 h57 minutes sur France 3, 79h01 minutes sur France 2, 5 h24 minutes sur M6 ${ }^{1}$. Les obligations de service public jouent ici un rôle décisif mais non exclusif. Malgré Michel Field puis Ruth Elkrieff, TF1 n'est parvenu à maintenir ni l'audience, ni un haut niveau d'invitations politiques (présidentiables et principaux ministres) qu'autorisait le dispositif historique plusieurs fois renouvelé de 7 sur 7 le dimanche à $19 \mathrm{~h}$, face à la concurrence de Michel Drucker lançant à son tour des invitations politiques avec succès.

1 Source: CSA, Rapport d'activité 2004. Avec 22h58 minutes hors JT, Canal Plus s'avère ainsi être de loin la chaîne privée qui consacre le plus de temps d'antenne à la parole politique : le constat vaut y compris pour le seul temps de parole politique au sein des journaux télévisés soit 8h41 minutes sur TF1, 36 h03 minutes sur France 2 et 16h44 minutes sur France 3. 
- Perdurent dans les formats classiques mais sur le service public. France 3 avec France-Europe Express depuis 1997, tout comme France 2 avec 100 minutes pour convaincre dirigé par Olivier Mazerolle à partir de la rentrée 2001 (mensuelle en prime time) font alterner les questionneurs journalistes selon leur domaine de compétence sur le modèle canonique de L'heure de vérité. Mots croisés avec Arlette Chabot sur France 2 et Ripostes sur France 5 sous la responsabilité de Serge Moati reprennent, eux, le modèle originel du « duel » initié par À armes égales en 1973. Toutes proposent systématiquement des entretiens journalistiques (le modèle américain d'origine du «face à la presse ») mais consacrent simultanément le retour à une autre formule conversationnelle - présent depuis les origines de la politique télévisée dans les années 50 - face à un autre homme politique («face à face »). Le format de la discussion avec des dépositaires reconnus (à tort ou à raison) du savoir («face aux experts ») se raréfie (il se retrouve que dans Ripostes) ; il semble avoir été remplacé par le questionnement des citoyens anonymes ( « face à l'opinion »). Les configurations électorales mais aussi l'acmé des «affaires » (parce que le système politique est alors lui-même perçu comme fragilisé) servent inévitablement la rédaction dans leur lutte quasi-quotidienne en interne contre les animateurs extérieurs à la chaîne : dans ces périodes, ces derniers se voient alors clairement interdire l'invitation d'hommes politiques' ${ }^{1}$.

1 Entretien informel avec un haut responsable de la rédaction de France 2. La société des journalistes de la rédaction de France 2 a d'ailleurs rendu public un texte explicite et pourtant peu repris dénonçant le «monarcho-populisme » lors de l'émission spéciale sur le Référendum prévoyant un questionnement du Président de la république par Patrick Poivre d'Arvor, un panel de « jeunes » et trois animateurs non issus des rédactions : «Ainsi, le président de la République a choisi : sa chaîne, ses interlocuteurs, ses animateurs, ses thèmes de débat. Le spectacle peut commencer, rien ne sera laissé au hasard : l'Elysée, sous la houlette de Claude Chirac et du grand chambellan de TF1, Robert Namias, a tout verrouillé depuis un mois. Cette rencontre veut mettre en scène un président «jeune et sympa» dans un «dialogue direct» avec les Français [...]. Cette pratique «d'adoubement» fait sourire nos voisins européens. Mais, aujourd'hui, les choses sont plus graves, elles révèlent l'aboutissement d'une logique mêlant l'information au spectacle. Ségolène Royal «chez» Ruquier, sur les pas de son camarade Mélenchon, Barnier ou Sarkozy «chez» Drucker, cette logique entérine progressivement la disparition d'une expertise sur la politique à la télévision. Ces mêmes hommes politiques, si prompts à dénoncer le populisme et la démagogie, se précipitent devant «les vrais gens de la vraie vie» pour y dévoiler leur vie privée, et accessoirement leur vision politique... La démocratie ne gagne rien à ce jeu qui nourrit le vote extrémiste et l'abstention [...] », Libération, 14 avril 2005, p. 5. 
L'hypothèse d'une reproduction à l'identique est tout aussi irrecevable que celle de la révolution copernicienne. Désormais, les programmes politiques à la télévision :

- Elargissent l'éventail de leurs invités au personnel politique de second rang auparavant invisible à la télévision : députés européens, députés, sénateurs, ministres de second rang, représentants de groupes d'intérêts, principaux élus locaux. Sauf exceptions rarissimes, il ne s'agit toutefois que des représentants des formations politiques dominantes (s'agissant des chaînes hertziennes analogiques, les représentants de tous les partis non représentés au parlement se partagent moins de 3\% du temps d'antenne politique total). Néanmoins, ces nouvelles arènes se développent exclusivement sur les nouvelles chaînes d'informations qui visent les CSP+, en particulier $L C I$ (avec ses cinq magazines économiques et ses quatre magazines d'entretiens politiques simultanés : Le grand jury, Questions qui fâchent, L'invité de PierreLuc Séguillon, Politiquement show). Pour des raisons d'efficacité politique et d'économies d'échelle (financement du sondage d'opinion qui supporte l'émission) les magazines politiques à la télévision ont souvent en réalité été multimédias (Grand Jury RTL-Le Monde, devenu RTL-LCI-Le Figaro, partenariat avec la presse magazine d'information générale...), mais cette tradition relève désormais de l'obligation avec la multiplication des chaînes fondée sur l'économie du narrowcast qui suppose de s'associer pour trouver les fonds nécessaires au financement de produits culturels, dont ceux politiques, à destination de niches d'audiences intéressées à l'information générale, économique et politique (le retour sur investissement est facilité par le fait qu'il s'agit en l'occurrence des CSP+ dotés de forts pouvoirs d'achat et très attractifs pour certains annonceurs mais les difficultés économiques demeurent bien réelles), d'où l'association par exemple de $L C I$ et $B F M$ autour du magazine $100 \%$ politique.

- Se diluent au sein d'autres émissions du programme ${ }^{1}$ dont Vivement Dimanche animé par Michel Drucker sur France 2 le dimanche après-midi pour 3 à 5 millions de téléspectateurs, On peut pas plaire à tout le monde avec Marc-Olivier Fogiel sur France 3, Tout le monde en

1 Erik Neveu, « De l'art (et du coût) d'éviter la politique. La démocratie du talk show version française (Ardisson, Drucker, Fogiel) » et « Le chercheur et l'infotainment : sans peur mais pas sans reproche. Quelques objections à la critique d'une imaginaire orthodoxie critique », Réseaux, ${ }^{\circ}$ 118, 2003, p. 95-134 et p. 167-182. Et l'ensemble de ce numéro « La politique saisie par le divertissement ». 
parle produit par Thierry Ardisson sur France 2 le dimanche soir ou Le vrai journal de Karl Zéro sur Canal Plus le dimanche à 12h40. Autant d'émissions qui accueillent régulièrement des interviews d'hommes politiques professionnels et contribuent effectivement à institutionnaliser l'interview politique par un animateur ${ }^{1}$.

Mais une triple précaution s'impose. D'abord, la frontière est plus que poreuse, mais la carte de presse n'a jamais été requise pour interroger les «puissants » (les speakerines s'en chargent occasionnellement dans les années 60 , dans un dispositif toutefois très particulier) et à l'inverse les journalistes politiques les plus en vue ont régulièrement bénéficié de fiches de paye en provenance des sociétés de production (Christine Ockrent avec Plaisance Film) ou ont même animé une émission de divertissement (Anne Sinclair ou Guillaume Durand). Ensuite, aujourd'hui encore et selon le CSA, la majeure partie du temps de parole politique se situe dans les «émissions d'information » précitées, celles directement gérées par la rédaction des chaînes. Enfin, et surtout, seul un regard myope ne tenant pas compte de la qualité sociale du recrutement et du questionnement peut confondre ces programmes pourtant très différents.

L'émission de Michel Drucker se distingue nettement par ses conditions de production (le questionnement est peu risqué) et de réception (l'audience peut dépasser les cinq millions de téléspectateurs) et, en conséquence, par la qualité sociale de son recrutement politique puisqu'elle peut faire valoir l'invitation de pratiquement tous les plus hauts responsables politiques nationaux à l'exception du Président de la République, toutefois « représenté » par son épouse Bernadette Chirac. C'est ainsi que l'émission de Michel Drucker occupe aujourd'hui du point de vue de ses invitations politiques la position du magazine politique de référence (dans la filiation de Cartes sur tables, 7 sur 7, L'heure de vérité, puis Questions à domicile). $82 \%$ des invitations dans ces magazines politiques de référence était réservées de fait au personnel politique de premier rang (premier ministre, ministres et anciens minis-

1 Il reste bien sûr difficile de fixer les seuils au sens de Genette, bien d'autres émissions $\mathrm{du}$ programme peuvent accueillir plus occasionnellement des hommes politiques, dont On a tout essayé de Laurent Ruquier à $19 \mathrm{~h}$ tous les jours, La méthode Cauet sur TF1, 20 h10 pétante avec Stéphane Bern sur Canal plus, etc. 
tres, chefs des principaux partis) ${ }^{1}$ et Vivement Dimanche atteint un niveau de sélectivité comparable².

À l'inverse, les autres émissions, dont les magazines politiques des rédactions (Mots croisés, Les 4 vérités...), notamment lorsqu'elles (et parce qu'elles) prévoient un duel avec un autre homme politique (comme À armes égales dans les années 70 qui déjà ne parvenait pas à obtenir l'invitation des leaders du champ politique), doivent se contenter plus souvent de personnalités politiques de second plan. Malgré le changement de chaîne, de dispositif et d'horaire, Vivement Dimanche apparaît bel et bien comme le continuateur du magazine Questions à domicile, dont il occupe la position de tribune politique de référence au profit exclusif (ou presque) des plus haut responsables du champ politique. Si ce «mélange des genres » n'a donc que l'apparence de la nouveauté, il prend cependant une dimension nouvelle, car Michel Drucker, non seulement évite, mais fustige les sujets politiques. La promotion des animateurs, qui ne signifie pas expropriation des journalistes politiques on l'a vu, est en réalité inséparable de la nouvelle économie de la production télévisuelle qui réussit aux professionnels qui ont su acquérir des compétences de chef d'entreprise. La spécialisation des chaînes sur leur métier de diffuseur entraîne une généralisation de la sous-traitance en matière de production télévisuelle et privilégie des savoir-faire polyvalents, encore inhabituels chez les journalistes de télévision. Le responsable d'une émission doit prendre en charge le projet depuis sa gestation ; s'investir personnellement et investir financièrement; négocier le projet «clés en main », autrement dit dans le jargon indigène P.A.D (Prêt À Diffuser), avec d'éventuels co-producteurs (trouver des sponsors) et avec la direction des chaînes (autant de tractations «honteuses » parce que liées à l'argent et nécessairement éloignées des préoccupations «nobles» de l'orthodoxie journalistique). Ces émissions du «mélange des genres » ne sont précisément pas celles de la rédaction mais les émissions sous-traitées gérées directement en face à face avec la direction de la chaîne. Depuis les pionniers Philippe Gildas, Michel Drucker ou Thierry Ardisson et jusqu'à

1 Eric Darras, «Media Consecration of the Political Order » in Rod Benson and Erik Neveu, eds, Bourdieu and the Journalistic Field, Cambridge, Polity Press, 2005, p. 162.

$211 \%$ de seconds rôles dans l'émission de Michel Drucker, 50\% chez Marc-Olivier Fogiel et 79\% chez Thierry Ardisson selon Aurélien Le Foulgoc, repris par Erik Neveu, art. cité, p. 113. 
Henry Chapier, Karl Zéro, Marc-Olivier Fogiel ou Jean-Luc Delarue. À l'exception relative de Thierry Ardisson mais aussi semble-t-il de Marc-Olivier Fogiel, les animateurs qui invitent les hommes politiques ne dissimulent que leurs ambitions relèvent moins du journalisme que de l'entertainment, à l'instar du pionnier Henry Chapier qui convient que pour Le Divan «le contenu et la démarche sont honnêtes, je les ai toujours valorisés $»^{1}$.

\section{Mise en scène de la vie privée}

Les mises en scène des espaces privés de l'invité politique ne sont toutefois ni nouvelles, ni imposées, ni mêmes désintéressées. Car l'exhibition télévisuelle du privé de l'homme politique de premier rang réside principalement dans une triple collusion d'intérêts entre ces associés/rivaux que sont les professionnels de la politique et de la télévision. D'abord, celui du volume et de la qualité sociale de l'audience rejoint les préoccupations électorales des élus (intéressés non seulement par un public massif mais aussi par les électeurs d'accès difficile, dont les jeunes urbains diplômés - amateurs de talk-shows « décontractés »-, soit ces primo-votants particulièrement stratégiques dans les élections disputées). Ensuite, la confrontation télévisuelle de l'homme politique et de l'électeur anonyme, tout comme la publicité télévisuelle de ses espaces privés, humanisent l'homme et la vie politiques. Selon un principe élémentaire de légitimation de la domination politique, le chef et le système politiques sont réputés grandis par les naturalisations paternalistes et/ou vertueuses (la vertu du bon père ou de la mère de famille ; l'authenticité des valeurs appliquées dans le cercle familial, le savoir pratique de l'élu qui est aussi un élu local...) ; le mérite du savoir (objectivé par les diplômes toujours très valorisés y compris dans les émissions de divertissement: ENA, Science Po, etc.) et de l'élection (légitimation par le nombre) ne semblant plus suffire. Enfin, le questionnement est assurément plus aisé et les « ratés » sont aussi rares que valorisés tant par la critique de la presse écrite que par la littérature d'analyse ${ }^{2}$. L'existence même de ces dispositifs peut être recherchée

1 Entretien téléphonique avec H. Chapier, 21 décembre 1994.

2 Les quelques rares chercheurs, dont Erik Neveu, qui ne se contentent pas d'écrire des articles en reprenant, à la manière des journalistes eux-mêmes, la revue de presse de l'émission, ne peuvent que constater que «l'échantillon analysé ne permet en rien de décrire ces programmes comme des guet-apens organisés pour discréditer le 
dans la nécessité revendiquée d'une alternative au profond désintérêt des Français pour la politique ${ }^{1}$ et a fortiori pour les émissions politiques de télévision réputées «invendables $»^{2}$. Inscrire publiquement l'élu dans ses espaces privés (son for privé avec Le divan ou son intérieur domestique avec Questions à domicile ou Vivement Dimanche), c'est lui reconnaître une existence d'homme ordinaire : il s'agit de réparer le lien politique entre gouvernés et gouvernants, comme le proclame en toute bonne foi démocratique Michel Drucker. À titre plus personnel, les spécialistes du marketing politique appliquent ici l'un des trois mécanismes de projection du lecteur dans le personnage du livre lu, tels qu'analysés par Robert Scholes : le chef politique doit être alternativement et selon les configurations politiques ou les types de publics visés, idéalisé ( «il est meilleur que nous », stratégie d'héroïsation en période de crise, de mise en avant des diplômes ou des qualités gestionnaires...) et humanisé («il est comme nous », c'est la mise en scène des espaces privés, etc.) $)^{3}$. Pour signifier l'homme ordinaire dissimulé derrière le «grand » homme politique, la télévision et les conseillers en communication politique ont très tôt convoqué des symboles appropriés : le domicile, l'enfance, la famille, les vacances et les loisirs de l'homme politique.

La première intrusion de la télévision au « domicile » d'un homme politique remonte à 1953 , lorsque le président du Conseil Joseph Lainiel invite Pierre Sabbagh et la télévision à l'hôtel Matignon. Le

\footnotetext{
personnel politique » (art. cité : p. 128), que «la présence de cadres d'injustice ou d'action collective (au sens de Gamson) est rare » (p. 178) « il paraît empiriquement assez difficile de leur associer un apport d'information très significatif sur les règles, enjeux, logiques sociales propres à l'univers politique » et au total, elles «entretiennent et banalisent des postures dépolitisées (en particulier celles des jeunes téléspectateurs diplômés et "branché-cyniques », en surplomb «radical chic », « revenus de tout sans avoir bougé ») » (p. 132) bref «leur contribution à une meilleure intelligence des échelons spatiaux, processus et acteurs effectifs de processus de décision qui affectent l'existence quotidienne des citoyens est à peu près nulle » (p. 178)

1 Daniel Gaxie, Le cens caché, Seuil, 1978.

2 Brigitte Le Grignou et Erik Neveu, «Emettre la réception : préméditations et réceptions de la politique télévisée », Réseaux, 1993, Hors série, p. 65-98.

3 Robert Scholes, «Les modes de la fiction » dans Genette (Gérard) et alii, Théorie des genres, Paris, Seuil, Points, 1986 (1977), p. 77-88. Par contre, le troisième registre de projection du lecteur dans le personnage de fiction ne fonctionne guère en politique, c'est celui de la pitié ( « il est pire que nous ») peu susceptible de crédibiliser l'image d'un leader politique.
} 
magazine politique de télévision qui s'invente au milieu des années 60 porte rapidement son intérêt sur la vie privée des hommes politiques, ce qui peut provoquer l'étonnement des journalistes de presse écrite. Jacques Siclier commente ainsi le premier Face à la presse consécutif à mai 1968 (toutefois diffusé six mois après les «événements») qui, précise-t-il, l'a «déçu» : «Et tout d'abord les questions touchant le domaine privé de la vie du Premier ministre n'avaient que faire dans un débat aussi rigoureusement minuté : "Monsieur le Premier ministre, qu'est-ce qui vous attire dans le golf ? M. le Premier ministre, aimezvous la peinture abstraite ?" La belle jambe que ça lui fait, au citoyen !» ${ }^{1}$. Sur le modèle des quelques documentaires rachetés aux télévisions américaines $^{2}$, Pierre Desgraupes présente le 21 avril 1970, une émission intitulée Adresse Elysée, «film exceptionnel en France, retraçant la vie familiale du chef de l'Etat, chose courante à l'étranger... ce film n'est pas politique $»^{3}$. Le téléspectateur peut y voir le président de la République se promener en tricot de laine, raviver le feu de cheminée, se moucher, prendre son petit-fils dans les bras... L'importation des living-rooms politics se poursuit jusqu'à la diffusion en mars 1985 des 24 éditions politiques de Questions à domicile, sur le modèle semblet-il d'une émission américaine déjà ancienne produite par E. Murrow, Himself, mais au titre d'une concession à sa chaine acceptée en échange du maintien de son magazine d'investigation. La séquence «intimité de l'homme politique » avec la visite pré-enregistrée du domicile y est néanmoins brève, encadrée et « très travaillée ${ }^{4}$.

La vie privée, c'est aussi la famille réunie autour de «l'heureux élu », sur le modèle de Jean Lecanuet aux présidentielles de $1965^{5}$. L'exemple n'a jamais cessé d'être suivi, y compris par les époux Bérégovoy sur le plateau de Patrick Sabatier, Stars 90. Les vacances des hommes politiques constituent un autre leitmotiv de la télévision française, tout comme leur enfance évoquée visuellement dès 1970 dans

1 Télérama, 6 octobre 1968.

2 Documentaires axés sur la vie privée de R. Nixon (16/01/1969) ou de N. Khrouchtchev («Une retraite bien tranquille», 28/08/1967) diffusés par le magazine Panorama

3 Introduction de Pierre Desgraupes selon le script de l'INA.

4 Brigitte Le Grignou et Erik Neveu, «Intimités publiques. Les dynamiques de la politique à la télévision», Revue française de science politique, vol 43, ${ }^{\circ} 6$, décembre 1993, pp. 940-969

5 Convaincu de l'incidence du mécanisme d'identification-projection des téléspectateurs, le slogan des affiches de Jean Lecanuet en 1965 est : «En famille, écoutez Jean Lecanuet à la TELEvision» 
À Armes égales qui improvise une biographie en image de ses invités conçue notamment à partir de l'album de famille de l'homme politique. La formule est reprise in extenso, vingt-cinq ans plus tard, par un autre magazine politique, Face à la 3, puis par Questions à domicile et $L a$ preuve par 5 avec Pierre-Luc Séguillon sur La Cinq. Quelques temps auparavant (1983), Aи cœur du débat sollicite un court autoportrait en image pour chacun des deux invités. Ces exhibitions télévisuelles de la vie privée obligent depuis l'origine au portrait hagiographique à la télévision plus encore que dans la presse écrite ${ }^{1}$.

À la télévision, la présentation de soi des talking-heads consacre par ailleurs l'importance politique et stratégique du visage et donc l'impératif d'autocontrôle du for intérieur, l'espace le plus privé de l'homme politique. Car c'est sur le visage, cette (sur)face signifiante du corps, que s'extériorise le plus lisiblement ce qui est vécu (ou faussement vécu) à l'intérieur du corps : la joie, la colère, l'émoi, l'angoisse... La caméra confère au visage une importance cruciale. Mais les gros plans, voire les très gros plans, existent depuis toujours à la télévision française. Les exigences du média-télévision n'ont fait au mieux que renforcer les mutations de l'éloquence politique. Le théâtre de la politique n'est certes plus la Cour, ce « laboratoire de la vie politique moderne » selon $\operatorname{Elias}^{2}$, mais bien les plateaux de télévision. Reste que l'autocontrôle des passions est un impératif de la rhétorique corporelle de l'homme politique depuis fort longtemps : qui pourrait prétendre gouverner l'Etat, et a fortiori les hommes, s'il ne sait gouverner son $\operatorname{corps}^{3}$ ? Lamartine s'entraînait déjà à haranguer devant son miroir, les «petites phrases » préméditées sont faussement improvisées depuis les discours révolutionnaires au moins. À l'occasion, la spontanéité des réactions émotives face aux questions, notamment personnelles, des journalistes, authentifie la sincérité de l'homme politique. Lorsque, dans L'heure de vérité, Laurent Fabius est interrogé par Albert du Roy au sujet de l'assassinat de son ami Jacques Perrot, le premier ministre « devient blême. Esquisse de la main comme un geste d'impuissance, ou de rage. Tourne la tête, cache son regard. Et enfin, les larmes aux yeux, répond d'une voix brisée : "quelle importance? Jacques Perrot

1 Legavre (Jean-Baptiste), «Un genre métis : le portrait de presse », dans Legavre, dir., Presse écrite : objets délaissés, Paris, L'harmattan, pp. 211-246.

2 Norbert Elias, La société de cour, Paris, Flammarion, 1985 (1933).

3 Claudine Haroche, «Se gouverner, gouverner les autres », Communications, n58, 1993, p. $51-69$ 
était mon ami depuis vingt-cinq ans, mon plus proche ami. Je voudrais simplement que dans tout le bruit qui l'entoure, on pense un peu plus à lui, qui est mort, et au chagrin de ceux qui l'aimaient $»^{1}$.

Par ailleurs l'histoire politique fourmille d'usages stratégiques, éventuellement ex post, du surgissement des émotions de responsables politiques, bien avant ou indépendamment de la télévision ${ }^{2}$. La professionnalisation est toutefois passée par là. On ne sait si la télévision relève du média «cool», mais le plateau de télévision reste lui en tout cas particulièrement « hot ». Pendant les directs, la chaleur diffusée par les projecteurs pose inévitablement le problème de la transpiration des invités. L'observation régulière des plateaux montre ainsi l'importance accordée au maquillage avant le direct puis les repoudrages systématiques des élus pendant les intermèdes ou hors champ. Les soins cosmétiques opérés contribuent à faire écran à l'ouverture des glandes sudoripares mais aussi à protéger par une pellicule souvent épaisse de fond de teint le surgissement éventuel des affects sur le visage de l'homme d'importance. Mais l'essentiel s'opère bien avant le direct, par une médication appropriée, par l'entrâ̂nement via les video-training ${ }^{3}$, mais aussi par la préparation de l'émission en amont avec ses responsables (à l'instar d'Anne Sinclair précédemment pour la préparation de Questions à domicile, Michel Drucker peut se rendre lui-même d'abord au domicile de son « invité » pour convenir avec lui des détails

1 Télérama, $\mathrm{n}^{\circ} 1879,15 / 01 / 1986, \mathrm{p} 50$

2 L'apparent stigmate peut ainsi être reconverti en emblème comme lorsque que la députée Christine Boutin, qui fond en larme après avoir brandi la bible dans l'hémicycle de l'Assemblée Nationale lors des débats sur le PACS, rend public le fait qu'elle a reçu le lendemain un bouquet en provenance d'une personne « haut placée » avant plus tard de publier un livre au titre explicite : Les larmes de la république. Un an plus tard, elle explique ainsi à Marion Van Reterghem pour Le Monde : « Ce n'était pas glorieux. Je me suis dit "Christine tu arrêtes, tu te ressaisis. Comment tu vas gérer ce truc". Puis j'ai vite compris que je pouvais en tirer des royalties. En disant que j'avais reçu le bouquet, je montrais du doigt l'erreur politique de Jospin ». Le Monde, 13 novembre 1999, p. 12.

3 Ces entraînements commencent désormais très tôt dans la carrière politique. En 2005 les 30es universités d'été des jeunes UDF prévoient des video trainings. Ce souci télévisuel de présentation de soi est toutefois né avec la télévision comme le remarque, parmi d'autres, Pierre Dumayet dès 1966 : Généralement, la personne qui va être interviewée à la télévision se prépare physiquement à l'être, elle va chez le coiffeur par exemple (...). La préparation de l'interview télévisuelle consiste essentiellement à préparer l'interviewé, c'est-à-dire à lui faire oublier son visage, l'inviter à privilégier ce qu'il veut dire sur ce que, spontanément, il veut paraître ", Communications, $\mathrm{n}^{\circ} 7,1966$. 
de sa future prestation télévisuelle. Erik Neveu précise qu'Edouard Balladur a conditionné sa présence sur le plateau de Thierry Ardisson à une communication préalable des questions de l'animateur ${ }^{1}$. C'est surtout au travers des dispositions charismatiques héritées et d'une aisance sociale caractéristique des familles les plus privilégiées socialement dont sont issus la quasi-totalité des élus nationaux en France, mais aussi de facilités acquises par la pratique répétée du discours public, dont les « grands » oraux réussis des « grands concours », que les principaux professionnels de la politique s'adaptent sas difficulté majeure à la télévision, comme à toutes sortes de tribunes obligées.

\section{Le sommet des années 80}

On peut convenir avec Erik Neveu, Dominique Mehl, Guy Lochard et Jean-Claude Soulages que la crise télévisuelle atteint son summum à la fin des années 80 . La décrue relative de «l'infotainment», tant en nombre d'émissions ou d'événements qu'en intensité dramatique, commence avec les années 90, notamment avec la faillite de La Cinq et de son magazine politique Les absents ont toujours tort animé par Guillaume Durand ${ }^{2}$. C'était aussi l'époque où quelques professionnels de la politique venaient en couple livrer des secrets d'alcôves sur le plateau de Tournez-Manège mais il est vrai dans une séquence distincte d'entretien avec Jean Amadou : Huguette Bouchardeau, Hervé de Charrette, Edith Cresson, Jean-Jacques Descamps, Hugues Dewarin, Jack Lang, Louis Mermaz, Roland Nungesser Michel d'Ornano, Georges Sarre. Il y a eu également les pleurs du ministre de la santé Michèle Barzach qui retrouve en direct, en gros plan et sous les applaudissements du public, son «nounou » marocain «perdu de vue » depuis plus de trente ans pour la séquence surprise de l'émission de Jean-Pierre Foucault, Sacrée Soirée. L'homme politique a encore pu directement être interrogé sur son «moi profond » à la télévision française avec Le Divan, une émission d'un quart d'heure diffusée après Soir 3 et lancée en 1987. Elle attire alors vingt d'hommes politiques dont quelques professionnels politiques de premier plan (Jack Lang, Alain Juppé, Simone Veil, Edith Cresson), mais les présidentiables sollicités déclinent systématiquement l'invitation. Henry Chapier confirme ainsi les refus de Bernard

\footnotetext{
Art. cité, p. 112.

Eric Darras, «Espaces privés à usages publics » dans Haroche (Claudine), dir., Le for intérieur, Paris, PUF-CURAPP, 1995, p. 378-397.
} 
Tapie, « en raison essentiellement de l'audience » ${ }^{1}$, de Valéry Giscard d'Estaing «sur les conseils de son entourage », de Raymond Barre, de François Mitterrand ou de Jacques Chirac que Henry Chapier a pourtant connu «à science po ». Depuis, l'insistance médiatique sur quelques cas aussi rares que cités peut tendre à faire passer les exceptions pour la règle.

Quoi qu'il en soit, aujourd'hui comme hier, les leaders politiques qui acceptent de participer aux émissions les plus insolites comptent parmi les profils les plus atypiques du champ politique, dont André Santini, Bernard Tapie ou Jack Lang (ce dernier fait figure de quasiidéal type : il accorde une «interview hard» dans Supersexy le 16 septembre 1987 comme il confirme, 18 ans plus tard, sa candidature à l'élection présidentielle dans un entretien accordé au magazine Voici, le 18 juillet 2005) ou occupent eux-mêmes des positions d'outsiders au sein de leurs propres partis (Pierre Juquin, Laurent Fabius depuis plusieurs années) ou au contrebas du gouvernement (secrétaires d'Etat ou ministres délégués plutôt que ministres et a fortiori « grands ministres avec motards » pour reprendre une distinction indigène) et du champ politique national (députés, élus locaux ${ }^{2}$ ). Ils semblent se situer plus souvent dans un parti-cadre au sens duvergien, en particulier au centre droit, qui autorise une plus grande autonomie de l'élu vis-à-vis de ses mandants et des ressources collectives du parti (à l'exact opposé du parti communiste français longtemps lié par les logiques représentatives de la délégation politique et qui répugna en conséquence à prendre part au nouveau jeu télévisuel, Robert Hue marquant le changement de ton). Beaucoup ont d'ailleurs échoué dans leur ascension politique au sommet de l'Etat rappelant peut-être ainsi le caractère biface de la légitimation télévisuelle qui peut être assimilée à la «superficialité », aux « paillettes », à la non-représentativité, au discours creux et qui ne se convertit que très imparfaitement en capital politique dans un univers aussi autonome que celui de l'élite politique. Ce n'est qu'au travers d'illusions rétrospectives que l'on attribue à ces « modernes » une réus-

1 Audience traditionnellement faible des programmes de fin de soirée : en sept ans, l'audimat oscille entre 200000 et 2 millions de personnes soit un rapport de 1 à 10 , en fonction notamment de l'heure de début de programmation qui varie entre $11 \mathrm{~h}$ et Oh.

2 Ce que confirme, pour le cas français, le décompte statistique d'Aurélien Le Fougoc, «1990-2002 : une décennie de politique à la télévision française », Réseaux, 118, op. cit, p. 56. 
site exemplaire, et rares sont ceux qui depuis le milieu des années 80 ont tenu la longueur.

En matière télévisuelle, les échecs renseignent au moins autant que les succès. Ils fixent les limites à ne pas dépasser pour qui prétend institutionnaliser un magazine politique de premier ordre. Lorsqu'à la rentrée de septembre 2003, Etienne Mougeotte, vice-président de TF1, tente le lancement d'un "nouveau » concept d'émission politique en « immergeant 36 heures durant un leader politique dans une famille française, il doit rapidement renoncer faute de candidats ${ }^{1}$. Un échange de bons procédés doit s'instaurer entre professionnels de la politique de premier rang et professionnels de la télévision. Le concept originel de «l'immersion dans une famille ordinaire » pourrait d'ailleurs être attribué au président Giscard d'Estaing s'invitant à la table d'une famille ordinaire sous les caméras bienveillantes de la télévision française. À nouveau, le problème n'est pas tant d'opposer à la manière des critiques journalistiques ou essayistes de la télévision le privé et le public que de comprendre que les espaces privés de l'homme politique peuvent être filmés tant que lui et/ou ses conseillers y voient un intérêt politique, dont celui électoral. Car en dernier ressort, nul journaliste, nul patron de chaîne ne dispose du pouvoir d'exiger la présence sur son plateau de télévision d'un homme politique de premier rang. Ces derniers s'invitant plus qu'ils ne sont invités, aujourd'hui comme hier et pour des raisons qui ont également trait à leur propension à générer de l'audience : un Premier ministre en exercice réalise presque systématiquement des records d'audience.

Last but not least, les émissions politiques prévoient presque systématiquement le questionnement de l'invité politique par des citoyens anonymes. Initiée, non pas par des professionnels de télévision, mais par des conseillers en communication pour l'élection américaine de $1968^{2}$, le recours aux questions posées par des citoyens anonymes s'impose à la télévision française comme ailleurs, et particulièrement en période électorale. Des tentatives hybrides sont essayées régulière-

1 De la même manière Audit, une émission initiée par Paul Amar en 1993 où l'homme politique descend dans la rue et le métro pour rencontrer les passants est vite abandonnée, faute de candidats.

2 Roger Ailes, certes ancien producteur du Mike Douglas Show mais conseiller de Richard Nixon suggère ainsi au candidat de se soumettre aux questions d'un panel judicieusement sélectionné d'électeurs anonymes en excluant les journalistes. J. Mc Guiniss, The Selling of the President, 1968, New York : Trident, 1969. 
ment, en particulier sur France 5 avec Les 109 le dimanche à 15 h, une émission forum de Paul Amar où les questions sont posées par des étudiants de "Science Po». Citons encore Du fer dans les épinards, D'un monde à l'autre, J'ai rendez vous avec vous ou Quand je serai président en 2002. Les dispositifs s'avèrent fragiles et éphémères, les citoyens «ordinaires » ressortent désormais du passage obligé (en témoigne leur intégration systématique aux émissions du programme et aux émissions pré-électorales). Même s'ils se suivent sur les plateaux et ne se ressemblent pas, la fonction elle-même qui leur est assignée par les productions varie intentionnellement (divertissement, édification, participation $)^{1}$.

\section{Jeux ou enjeux}

Est-ce à dire que le niveau baisse inévitablement? Animateurs et citoyens lambda ne peuvent pourtant faire valoir, sauf exceptions, le professionnalisme et les compétences politiques des journalistes. Il reste difficile et délicat de prétendre jauger non plus la seule quantité de discours politique mais de sa «qualité ». Plusieurs travaux congruents s'y sont pourtant attelés avec un résultat surprenant. Une distinction désormais classique sépare le questionnement des hommes politiques par les journalistes ou autres (notamment les citoyens anonymes face aux leaders politiques sur les plateaux de télévision) selon qu'il porte sur le jeu (la compétition politique, horse race politics, le marketing, les petites phrases...) ou les enjeux politiques. Aux Etats-Unis, Dan Hallin montre que si le nombre d'occurrence de sujets consacrés au jeu politique augmente très sensiblement depuis 1968, il en va finalement de même du discours journalistique consacré aux enjeux à partir des années 80, moment d'une prise de conscience journalistique dont il attribue une part de responsabilité à la critique ${ }^{2}$.

L'analyse par Erik Neveu de cinq éditions du magazine La France en direct révèle que le questionnement de l'invité politique par les journalistes politiques porte dans $68 \%$ des cas sur « la course de petits chevaux » mais pour seulement $4 \%$ des questions des citoyens anonymes qui préfèrent interroger l'homme politique sur les sujets qui concernent le plus

1 Pierre Lefébure, Quand les citoyens discutent le lien représentatif, Thèse de doctorat de science politique, IEP de Paris, 2005.

2 Dan Hallin, We keep America at the Top of the World, London and New York: Routledge, 1994, p. 145. 
grand nombre en l'occurrence le chômage, l'Etat providence et l'éducation $^{1}$. En reprenant cette grille d'analyse, Thomas Isle de Beauchaine montre que les questionnements politiques ne se résument pas à l'opposition distinguant « journaliste politique » et « citoyens anonymes ». Ainsi, le questionnement par la journaliste Françoise Laborde ne porte pratiquement jamais sur la horse race politics dans la séquence matinale de Télématin, Les 4 vérités, mais compte pour $21 \%$ des questions de Questions ouvertes tandis que $76 \%$ des questions des étudiants anonymes de Science Po Paris à Jean-François Copé portent sur la horse race politics ou sur la personnalité, le style de vie et les goûts de l'invité dans l'émission Les 109 animée par Paul Amar². C'est le paradoxe par lequel le degré de réalité des enjeux traités dans les émissions politiques (la «qualité » du questionnement) - chômage, précarisation, éducation, santé, baisse du pouvoir d'achat, insécurité... - s'accroît à mesure que le questionneur s'éloigne du champ politique. Les insiders, dont les journalistes issus des écoles et de Science Po Paris comme leurs interlocuteurs politiques, et a fortiori les étudiants de Science Po Paris, posent et se posent des questions formées au moule des interrogations propres aux acteurs et institutions politiques dominants (qui sera le $3^{\mathrm{e}}$ homme ? qui va gagner? la candidature à la candidature ? la déconstruction du marketing politique, etc. relèvent de questions d'insiders largement déconnectées des attentes réelles des citoyens). À l'inverse, une femme journaliste formée à l'Université (par opposition aux Ecoles) et aux Etats-Unis (Françoise Laborde dans Les 4 vérités), et de surcroît libérée des contraintes « diplomatiques » faute d'une audience stratégique, peut parfaitement, comme les citoyens anonymes sur les plateaux, promouvoir un questionnement plus axé sur le réel des Français parce que plus distant des préoccupations politiciennes.

Le questionnement politique des citoyens les plus démunis s'appuie, comme le montre William Gamson notamment, sur une connaissance pratique, sur l'expérience vécue et la discussion collective (fermeture d'usines, d'hôpitaux, chômage, insécurité réelle, etc.) $)^{3}$ ou sur une forma-

1 Erik Neveu, «Des questions «jamais entendues». Crise et renouvellements du journalisme politique à la télévision», Politix, 37, 1997, pp. 25-56, p. 54.

2 Ces chiffres sont à considérer avec précaution dans la mesure où ils ne portent que sur une période très limitée voire sur l'analyse d'une seule émission. Cf. Isle de Beauchaine (Thomas), La politique télégénique. Les dispositifs des émissions politiques en 2004, mémoire de fin de diplôme de l'IEP de Toulouse, 2004.

3 William A. Gamson, Talking politics, Cambridge, Cambridge University Press, 1992 
tion syndicale au sein de l'entreprise moins dépendante des croyances et valeurs partagées dans les partis politiques de gouvernement.

Changements et continuités coexistent donc à la télévision française. Parmi les explications avancées des permanences ou des mutations, il reste bien difficile de démêler la part respectives des facteurs de causalité. Du côté des forces de la conservation non pas à l'identique mais retouchée, citons la hausse du niveau culturel mais aussi le vieillissement des audiences des chaînes historiques, le contrôle du respect du pluralisme par le CSA en période électorale, la résistance en interne des rédactions nationales aidées par un syndicalisme encore puissant au sein du service public, l'absence de concurrence de la chaîne leader TF1 en matière politique mais surtout le refus de comparaître des plus hauts responsables politiques. Tout cela concourt au maintien des formats classiques d'émissions politiques, dont ceux mettant respectueusement en scène la vie privée des plus hauts responsables politiques. À l'opposé, la féminisation du journalisme politique ${ }^{1}$ (les espaces privés touchant au féminin...), la nouvelle économie des médias, l'affinement des indicateurs d'audience, la dérégulation hors période électorale, l'insuffisance chronique des financements par la redevance, les faiblesses du recrutement (la télévision constituant rarement le choix des meilleurs étudiants journalistes) comme de la formation des journalistes... mais aussi et peut-être surtout les transformations du champ politique lui-même (européanisation des décisions, exigences de démocratie participative, dispersion des formations politiques, concurrence intestine, polarisation aux extrêmes, montée en puissance des sondeurs et conseillers en communication, mais aussi des juges et des intellectuels, apparition de nouveaux entrepreneurs politiques porteurs de nouvelles causes, expropriation par l'économique...) contribuent aux changements moins radicaux qu'il y paraît en matière de politique télévisée. Reste la question des effets des ces mises en scène politiques sur les opinions et les comportements politiques des téléspectateurs qui est bien loin d'être réglée ${ }^{2} \ldots$

1 Erik Neveu, «Le genre du journalisme. Des ambivalences de la féminisation d'une profession», Politix, n51, 2000, pp. 179-212

2 Deux synthèses récentes des travaux sont désormais disponibles en français : Brigitte Le Grignou, Du coté des publics. Usages et réceptions de la télévision, Paris, Economica, 2003 ; Rémy Rieffel, Que sont les médias ?, Paris, Folio, 2005. 